\title{
A REMARK ON A CONJECTURE OF MARCUS ON THE GENERALIZED NUMERICAL RANGE
}

\author{
by YIK-HOI AU-YEUNG and KAM-CHUEN NG
}

(Received 10 April, 1982)

1. Introduction. Let $A$ be an $n \times n$ complex matrix and $c=\left(c_{1}, \ldots, c_{n}\right) \in \mathbb{C}^{n}$. Define the $c$-numerical range of $A$ to be the set $W_{c}(A)=\left\{\sum_{j=1}^{n} c_{i} x_{j} A x_{i}^{*}:\left\{x_{1}, \ldots, x_{n}\right\}\right.$ is an orthonormal set in $\left.\mathbb{C}^{n}\right\}$, where ${ }^{*}$ denotes the conjugate transpose. Westwick $[8]$ proved that if $c_{1}, \ldots, c_{n}$ are collinear, then $W_{c}(A)$ is convex. (Poon [6] gave another proof.) But in general for $n \geqq 3, W_{c}(A)$ may fail to be convex even for normal $A$ (for example, see Marcus [4] or Lemma 3 in this note) though it is star-shaped (Tsing [7]). In the following, we shall assume that $A$ is normal. Let $W(A)=\left\{\operatorname{diag} U A U^{*}: U\right.$ is unitary . Horn [3] proved that if the eigenvalues of $A$ are collinear, then $W(A)$ is convex. Au-Yeung and Sing [2] showed that the converse is also true. Marcus [4] further conjectured (and proved for $n=3$ ) that if $W_{c}(A)$ is convex for all $c \in \mathbb{C}^{n}$, then the eigenvalues of $A$ are collinear. Let $\lambda=\left(\lambda_{1}, \ldots, \lambda_{n}\right) \in \mathbb{C}^{n}$. We denote by $\bar{\lambda}$ the vector $\left(\bar{\lambda}_{1}, \ldots, \bar{\lambda}_{n}\right)$ and by $[\lambda]$ the diagonal matrix with $\lambda_{1}, \ldots, \lambda_{n}$ lying on its diagonal. Since, for any unitary matrix $U, W_{c}(A)=$ $W_{c}\left(U A U^{*}\right)$, the Marcus conjecture reduces to: if $W_{c}([\lambda])$ is convex for all $c \in \mathbb{C}^{n}$, then $\lambda_{1}, \ldots, \lambda_{n}$ are collinear. For the case $n=3$, Au-Yeung and Poon [1] gave a complete characterization on the convexity of the set $W_{c}([\lambda])$ in terms of the relative position of the points $\sum_{i=1}^{3} c_{j} \lambda_{\sigma(j)}$, where $\sigma \in S_{3}$, the permutation group of order 3 . As an example they showed that if $\lambda_{1}, \lambda_{2}, \lambda_{3}$ are not collinear, then $W_{\bar{\lambda}}([\lambda])$ is not convex (Lemma 3 in this note gives another proof). We shall show that for the case $n=4, W_{\bar{\lambda}}([\lambda])$ is not convex if $\lambda_{1}, \lambda_{2}, \lambda_{3}, \lambda_{4}$ are not collinear. Thus for $n=3,4$ the Marcus conjecture is answered and improved.

2. The convexity of $c$-numerical range. A real nonnegative $n \times n$ matrix $S=\left(s_{i j}\right)$ is said to be doubly-stochastic if every row and column sum of $S$ is 1 and orthostochastic (o.s.) if there exists a unitary matrix $U=\left(u_{i j}\right)$ such that $s_{i j}=\left|u_{i j}\right|^{2}, i, j=1, \ldots, n$.

The following two lemmas are obvious.

LEMMA 1. $W_{\mathrm{c}}([\lambda])=\left\{\lambda S c^{T}: S\right.$ is o.s. $\}$, where $T$ denotes the transpose.

Lemma 2. Let $\mu=\alpha \lambda+\beta(1,1, \ldots, 1)$, where $\alpha, \beta \in \mathbb{C}$ and $\alpha \neq 0$. Then, for any $c \in \mathbb{C}^{n}$, $W_{c}([\lambda])$ is convex if and only if $W_{c}([\mu])$ is convex.

We shall denote $W_{\bar{\lambda}}([\lambda])$ by $W(\lambda)$. Let $\mathscr{P}_{n}$ be the set of all $n \times n$ permutation matrices and $\mathscr{P}(\lambda)=\left\{\lambda P \lambda^{*}: P \in \mathscr{P}_{n}\right\}$. Then, by Lemma 1 and Birkhoff's Theorem (for example, see Mirsky [5]), we have $\mathscr{P}(\lambda) \subset W(\lambda) \subset$ convex hull of $\mathscr{P}(\lambda)$. Let $m(\lambda)=\lambda I \lambda^{*}$, where $I$ is the identity matrix. Then $m(\lambda)$ is positive if $\lambda \neq 0$ and is of largest magnitude

Glasgow Math. J. 24 (1983) 191-194. 
among points in $\mathscr{P}(\lambda)$. It is obvious that both $\mathscr{P}(\lambda)$ and $W(\lambda)$ are symmetric about the real axis. Suppose $\lambda_{1}, \ldots, \lambda_{n}$ are not collinear. Consider the lines joining a point in $\mathscr{P}(\lambda) \backslash\{m(\lambda)\}$ and $m(\lambda)$; we see that there are two such lines $L$ (in fact one is the reflection of the other about the real axis) such that all points in $\mathscr{P}(\lambda)$ lie on or on one side of $L$. For $n=4$, we shall show that if $m \in \mathscr{P}(\lambda) \backslash\{m(\lambda)\}$ is a point on $L$ which is nearest to $m(\lambda)$, then there are points in the open line segment $(m, m(\lambda))$ which are not in $W(\lambda)$. Before we prove our result, we need the following lemma.

LEMmA 3. If $\lambda=(1, \alpha, 0,0, \ldots, 0) \in \mathbb{C}^{n}$, where $\alpha$ is not real, then $W(\lambda)$ is not convex for $n \geqq 3$.

Proof. Obviously, we have $m(\lambda)=1+\alpha \bar{\alpha}$ and the non-real points in $\mathscr{P}(\lambda)$ are $\alpha$ and $\bar{\alpha}$. Let $V(\xi)=\left\{P \in \mathscr{P}_{n}: \lambda P \lambda^{*}=\xi\right\}$, where $\xi \in \mathbb{C}$. If $\left(p_{i \mathrm{j}}\right) \in V(\alpha)$, then $p_{11}=p_{12}=0$ and $p_{21}=1$ and if $\left(p_{i j}\right) \in V(1+\alpha \bar{\alpha})$, then $p_{11}=p_{22}=1$. Suppose that $0<t<1$ and $z=$ $(1-t) \alpha+t(1+\alpha \bar{\alpha}) \in W(\lambda)$. Then, by Lemma 1 , there exists an o.s. matrix $S$ such that $z=\lambda S \lambda^{*}$. By Birkhoff's theorem, $S$ is a convex combination of permutation matrices. Since $z$ is on the open line segment $(\alpha, 1+\alpha \vec{\alpha})$ and all other points in $\mathscr{P}(\lambda)$ lie on one side of the line joining $\alpha$ and $1+\alpha \bar{\alpha}$, we have

$$
S=(1-t)\left(\sum_{p \in V(\alpha)} t_{P} P\right)+t\left(\sum_{P \in V(1+\alpha \bar{\alpha})} t_{P} P\right),
$$

where $\sum_{P \in V(\alpha)} t_{P}=\sum_{P \in V(1+\alpha \bar{\alpha})} t_{P}=1$ and $t_{P} \geqq 0$ for all $P \in V(\alpha) \cup V(1+\alpha \bar{\alpha})$.

Considering the first two columns of $S$, we see that $S$ cannot be o.s. Hence $W(\lambda)$ is not convex.

THEOREM 4. Let $\lambda=\left(\lambda_{1}, \lambda_{2}, \lambda_{3}, \lambda_{4}\right) \in \mathbb{C}^{4}$. If $\lambda_{1}, \lambda_{2}, \lambda_{3}, \lambda_{4}$ are not collinear, then $W(\lambda)$ is not convex.

Proof. There are at most 24 points in $\mathscr{P}(\lambda)$ and since there are 10 symmetric permutation matrices in $\mathscr{P}_{4}$, there are at most 7 points in $\mathscr{P}(\lambda)$ lying on the upper (lower) half-plane. By Lemma 2 and Lemma 3, we may assume $\lambda_{1}, \lambda_{2}, \lambda_{3}, \lambda_{4}$ are distinct. Then $I$ is the only permutation matrix corresponding to $m(\lambda)$. Using Lemma 2 and the fact that $W(\lambda)=W(\bar{\lambda})$, we may assume $\lambda=\left(r_{1}, r_{2}, r_{3} e^{i \theta},-r_{4} e^{i \theta}\right)$ or $\lambda=\left(r_{1},-r_{2}, r_{3} e^{i \theta},-r_{4} e^{i \theta}\right)$, where $r_{1} \geqq 0, r_{2} \geqq 0, r_{3}>0, r_{4}>0$ and $0<\theta \leqq \pi / 2$ according as the convex hull of $\left\{\lambda_{1}, \lambda_{2}, \lambda_{3}, \lambda_{4}\right\}$ has 3 or 4 vertices.

Case 1. $\lambda=\left(r_{1}, r_{2}, r_{3} e^{i \theta},-r_{4} e^{i \theta}\right)$

In this case, for definiteness, we assume $r_{1}>r_{2}$. Then the 7 possible points in $\mathscr{P}(\lambda)$ and on the upper half-plane are:

$$
\begin{aligned}
& m_{1}=\lambda P_{1} \lambda^{*}=r_{3}^{2}+r_{1} r_{2}-\left(r_{1} r_{4}+r_{2} r_{4}\right) \cos \theta+i\left(r_{1} r_{4}-r_{2} r_{4}\right) \sin \theta, \\
& m_{2}=\lambda P_{2} \lambda^{*}=r_{2}^{2}-r_{3} r_{4}+\left(r_{1} r_{3}-r_{1} r_{4}\right) \cos \theta+i\left(r_{1} r_{3}+r_{1} r_{4}\right) \sin \theta, \\
& m_{3}=\lambda P_{3} \lambda^{*}=r_{4}^{2}+r_{1} r_{2}+\left(r_{1} r_{3}+r_{2} r_{3}\right) \cos \theta+i\left(r_{1} r_{3}-r_{2} r_{3}\right) \sin \theta, \\
& m_{4}=\lambda P_{4} \lambda^{*}=r_{1}^{2}-r_{3} r_{4}+\left(r_{2} r_{3}-r_{2} r_{4}\right) \cos \theta+i\left(r_{2} r_{3}+r_{2} r_{4}\right) \sin \theta,
\end{aligned}
$$




$$
\begin{aligned}
& m_{5}=\lambda P_{5} \lambda^{*}=\left(r_{1}+r_{2}\right)\left(r_{3}-r_{4}\right) \cos \theta+i\left(r_{1}-r_{2}\right)\left(r_{3}+r_{4}\right) \sin \theta, \\
& m_{6}=\lambda P_{6} \lambda^{*}=r_{1} r_{2}-r_{3} r_{4}+\left(r_{1} r_{3}-r_{2} r_{4}\right) \cos \theta+i\left(r_{1} r_{3}+r_{2} r_{4}\right) \sin \theta, \\
& m_{7}=\lambda P_{7} \lambda^{*}=r_{1} r_{2}-r_{3} r_{4}+\left(r_{2} r_{3}-r_{1} r_{4}\right) \cos \theta+i\left(r_{2} r_{3}+r_{1} r_{4}\right) \sin \theta .
\end{aligned}
$$

The permutations corresponding to the permutation matrices $P_{1}, P_{2}, \ldots, P_{7}$ are (142), (143), (123), (243), (1423), (1243), (1432) respectively. Let $m \in\left\{m_{k}: k=1, \ldots, 7\right\}$ be the point on $L$ which is nearest to $m(\lambda)$. Suppose that $0<t \leqq \frac{1}{2}$ and $z=(1-t) m(\lambda)+t m \in$ $W(\lambda)$; then using similar argument as in the proof of Lemma 3, there exists an o.s. matrix $S$ such that $z=\lambda S \lambda^{*}$ and

$$
\begin{aligned}
S & =t_{1} P_{1}+t_{2} P_{2}+\ldots+t_{7} P_{7}+t_{8} I \\
& =\left[\begin{array}{cccc}
t_{4}+t_{8} & t_{3}+t_{6} & 0 & t_{1}+t_{2}+t_{5}+t_{7} \\
t_{1}+t_{7} & t_{2}+t_{8} & t_{3}+t_{5} & t_{4}+t_{6} \\
t_{2}+t_{3}+t_{5}+t_{6} & t_{4}+t_{7} & t_{1}+t_{8} & 0 \\
0 & t_{1}+t_{5} & t_{2}+t_{4}+t_{6}+t_{7} & t_{3}+t_{8}
\end{array}\right]
\end{aligned}
$$

where $\frac{1}{2} \leqq 1-t \leqq t_{8}<1, \sum_{j=1}^{8} t_{j}=1$ and $t_{j} \geqq 0(j=1, \ldots, 8)$. Since $t_{8} \geqq \frac{1}{2} \geqq t_{7}$, from the first and the third columns of $S$, we have $t_{2}=t_{3}=t_{5}=t_{6}=0$ and then from the first and the second columns of $S$, we have $t_{1}=t_{7}=0$. Now columns 3 and 4 give $t_{4}=0$. This is a contradiction.

Case 2. $\lambda=\left(r_{1},-r_{2}, r_{3} e^{i \theta},-r_{4} e^{i \theta}\right)$

In this case we cannot use the method as in case 1 . We first have to eliminate two points in $\mathscr{P}(\lambda)$ and on the upper (closed) half-plane that cannot lie on $L$. Replace $r_{2}$ by $-r_{2}$ in $m_{k}(k=1, \ldots, 7)$ in case 1 and still denote them by $m_{k}$. Since we are considering points in the upper half-plane, we take $\bar{m}_{4}$ instead of $m_{4}$. If $r_{1} r_{3}-r_{2} r_{4}<0$, we take $\bar{m}_{6}$ and if $r_{1} r_{4}-r_{2} r_{3}<0$, we take $\bar{m}_{7}$. By comparing the slopes of the lines joining $m(\lambda)$ and $m_{5}$, $m(\lambda)$ and $m_{l}$ (or $\left.\bar{m}_{l}\right)(l=6,7)$, and by direct calculation, we see that $m_{6}, \bar{m}_{6}, m_{7}$ and $\bar{m}_{7}$ cannot lie on $L$. So the possible points in $\mathscr{P}(\lambda)$ and on the upper half-plane that lie on $L$ are $m_{1}, m_{2}, m_{3}, \bar{m}_{4}$ and $m_{5}$. Let $m \in\left\{m_{1}, m_{2}, m_{3}, \bar{m}_{4}, m_{5}\right\}$ be the point on $L$ which is nearest to $m(\lambda)$. Suppose $0<t<1$ and $z=(1-t) m(\lambda)+t m \in W(\lambda)$. Then, as in case 1 , there is an o.s. matrix $S$ such that $z=\lambda S \lambda^{*}$ and

$$
\begin{aligned}
S & =t_{1} P_{1}+t_{2} P_{2}+t_{3} P_{3}+t_{4} P_{4}^{T}+t_{5} P_{5}+t_{6} I \\
& =\left[\begin{array}{cccc}
t_{4}+t_{6} & t_{3} & 0 & t_{1}+t_{2}+t_{5} \\
t_{1} & t_{2}+t_{6} & t_{3}+t_{4}+t_{5} & 0 \\
t_{2}+t_{3}+t_{5} & 0 & t_{1}+t_{6} & t_{4} \\
0 & t_{1}+t_{4}+t_{5} & t_{2} & t_{3}+t_{6}
\end{array}\right],
\end{aligned}
$$
where $0<1-t \leqq t_{6}<1, \sum_{j=1}^{6} t_{j}=1$ and $t_{j} \geqq 0(j=1, \ldots, 6)$. From the first and the third
columns of $S$, we have

$$
\left(t_{2}+t_{3}+t_{5}\right)\left(t_{1}+t_{6}\right)=t_{1}\left(t_{3}+t_{4}+t_{5}\right)
$$


Since $t_{6}>0$, we have $t_{4} \geqq t_{2}$. From the second and the fourth columns of $S$, we have

$$
\left(t_{1}+t_{4}+t_{5}\right)\left(t_{3}+t_{6}\right)=t_{3}\left(t_{1}+t_{2}+t_{5}\right) \text {. }
$$

Since $t_{6}>0$, we have $t_{2} \geqq t_{4}$. Hence $t_{2}=t_{4}$. From (1) $t_{2}=t_{3}=t_{4}=t_{5}=0$. Now column 1 and 2 give $t_{1}=0$. This is a contradiction.

Hence in both cases, $W(\lambda)$ is not convex.

AdDEd IN Proof. Very recently Au-Yeung and Tsing by using less constructive method have proved that for arbitrary $n$ if the coordinates of $\lambda$ are not collinear, then there is a very small portion of the line $L$ which is not in $W(\lambda)$ and consequently have proved Theorem 4 for general $n$. This will be published in due course.

\section{REFERENCES}

1. Y. H. Au-Yeung and Y. T. Poon, $3 \times 3$ orthostochastic matrices and the convexity of generalized numerical ranges, Linear Algebra and Appl. 27 (1979), 69-79.

2. Y. H. Au-Yeung and F. Y. Sing, A remark on the generalized numerical range of a normal matrix, Glasgow Math. J. 18 (1977), 179-180.

3. A. Horn, Doubly stochastic matrices and the diagonal of a rotation matrix, Amer. J. Math. 76 (1954), 620-630.

4. M. Marcus, Some combinatorial aspects of numerical range, Ann. New York Acad. Sci. 319 (1979), 368-376.

5. L. Mirsky, Proofs of two theorems on doubly-stochastic matrices, Proc. Amer. Math. Soc. 9 (1958), 371-374. 35-37.

6. Y. T. Poon, Another proof of a result of Westwick, Linear and Multilinear Algebra 9 (1980),

7. N. K. Tsing, On the shape of the generalized numerical ranges, Linear and Multilinear Algebra 10 (1981), 173-182.

8. R. Westwick, A theorem on numerical range, Linear and Multilinear Algebra 2 (1975), 311-315.

Department of Mathematics

UNIVERSITY OF HONG KONG 